\title{
Steady-state moisture diffusion in curved walls, in the absence of condensate flow, via the BEM: a practical Civil Engineering approach (Glaser method)
}

\author{
António Tadeu*, Nuno Simões, Fernando Branco \\ Civil Engineering Department, Faculty of Sciences and Technology, University of Coimbra, Polo II - Pinhal de Marrocos, 3030-290 Coimbra, Portugal
}

Received 14 June 2002; received in revised form 13 September 2002; accepted 8 November 2002

\begin{abstract}
The influence of the curvature radius of curved walls on the condensation patterns across a single panel homogeneous wall is analysed under steady-state conditions. Condensation is defined according to the Glaser approach, which is a practical tool in building design, recommended by the DIN 4108 and prEN ISO 13788 standards. This methodology uses an iterative process, which requires the resolution of temperature equilibrium and several vapour pressure equilibrium problems. Each of these potential problems is solved using the Boundary Element Method (BEM). The iterative process is first implemented and validated by applying it to the definition of condensation patterns across a hollow cylinder, for which the solution is calculated analytically. The BEM is then applied to the curved wall models, identifying the zones where condensation occurs and quantifying the amount of liquid water generated.
\end{abstract}

(C) 2003 Elsevier Science Ltd. All rights reserved.

Keywords: Condensation; Temperature; Heat transfer; Vapour pressure; Moisture diffusion; Boundary Element Method

\section{Introduction}

Several methods have been proposed for studying condensation inside building elements, including those described by Glaser [1], Krischer et al. [2], Luikov [3], and Philip et al. [4]. The Glaser method is often used to predict condensation risks and to define the quality standards to be met by Civil Engineering construction elements. This method is even indicated by the DIN 4108 [5] and prEN ISO 13788 [6] standards to define condensation.

The Glaser method is based on the following assumptions: the transport of moisture obeys Fick's law; the heat is transported according to Fourier's law; the process occurs under steady-state conditions; the building elements are airtight, and condensed moisture cannot migrate. The material properties are assumed to be constant, even under the condensation conditions, which occur when the vapour pressure equals the vapour saturation pressure (the maximum vapour pressure allowed). Thus, the Glaser method only takes into account the vapour transport mechanisms and does not consider the effects of liquid transport.

\footnotetext{
* Corresponding author. Tel.: +351-239797201; fax: +351-239797190

E-mail address: tadeu@dec.uc.pt (A. Tadeu).
}

Most of the numerical models based on the Glaser approach are restricted to a simple one-dimensional mathematical formulation. This procedure is adequate if the element is straight, has constant thickness, is made of homogeneous material and is subject to uniform state conditions on both sides. There are situations, such as thermal bridges, where a one-dimensional formulation is not accurate enough. It should be noted that condensation is more likely to occur at the thermal bridges, since these involve materials whose thermal conductivity and moisture permeability properties differ.

A variety of numerical techniques have been used in these cases, among which are the Finite Elements (e.g. Bathe [7]) and Finite Differences methods (e.g. Freitas et al. [8]), which have been proposed to model and analyse the problem. These methods require the full discretization of the domain being studied, which entails highly expensive numerical computational schemes.

Away from a thermal bridge, the study of steady-state heat and moisture diffusion across homogeneous walls usually relies on a simple one-dimensional mathematical formulation. The same procedure is often used for curved walls of uniform thickness. However, this model may perform poorly if 


\section{Nomenclature}

\author{
e thickness, $\mathrm{m}$ \\ $\vec{g} \quad$ vapour flux, $\mathrm{kg} \mathrm{m}^{-2} \mathrm{~s}^{-1}$ \\ $h$ radiation and convection heat transfer coefficient, \\ $\mathrm{W}^{\circ} \mathrm{C}^{-1} \mathrm{~m}^{-2}$ \\ $P_{\mathrm{s}} \quad$ vapour saturation pressure, $\mathrm{Pa}$ \\ $P_{w} \quad$ vapour pressure, $\mathrm{Pa}$ \\ $\vec{q} \quad$ heat flux, $\mathrm{W} \mathrm{m}^{-2}$ \\ $r$ distance between the source and the internal point, \\ $\mathrm{m}$
}

$\begin{array}{ll}T & \text { temperature, }{ }^{\circ} \mathrm{C} \\ x, y & \text { rectangular coordinates, } \mathrm{m} \\ \text { Greek symbols } & \\ \lambda & \text { thermal conductivity, } \mathrm{W}^{\circ} \mathrm{C}^{-1} \mathrm{~m}^{-1} \\ \pi_{\mathrm{p}} & \text { vapour permeability, } \mathrm{kg} \mathrm{Pa}^{-1} \mathrm{~s}^{-1} \mathrm{~m}^{-1} \\ \text { Subscripts } & \\ \mathrm{e} & \text { external } \\ \mathrm{i} & \text { internal }\end{array}$

Greek symbols

$\pi_{\mathrm{p}}$

i internal the curvature of the wall is pronounced, in which case it may even lead to wrong hygrothermal analysis interpretations.

The present work analyses the alteration of condensation patterns across a homogeneous single-panel wall when a section of curved wall is placed between two perpendicular straight walls. It makes use of the Boundary Element Method (BEM). The BEM allows a compact description of the region, discretizing only the material discontinuities. Although the BEM leads to a fully populated system of equations, as opposed to the sparse system given by the Finite Difference and Finite Element schemes. The technique is efficient because it substantially reduces the size of the system of linear equations that needs to be solved. It is well known that the BEM uses the appropriate fundamental solutions, or Green's functions, to relate the field variables in a homogeneous medium to point sources placed within it. The fundamental solution most often used is that for an infinite homogeneous space, because it is known in closed-form and has a relatively simple structure. One of the drawbacks of the BEM is that it can only be applied to more general geometry and media when the fundamental solution is known, to avoid the discretization of the boundary interfaces, and this may not be possible.

The BEM has already been used to solve heat and moisture diffusion problems. Mingfang et al. [9] used the BEM to define the thermal characteristics of a column in contact with an insulated floor under steady-state conditions. Fratantonio et al. [10] calculated the required boundary element integrals analytically, using a different order of interpolation functions, to solve two-dimensional problems governed by the Laplace equation. Kassab et al. [11] have proposed a generalized boundary integral equation for isotropic heat conduction with spatially varying thermal conductivity. Ochiai et al. [12] used multiple-reciprocity analysis to analyse steady heat conduction. Melnikov [13] presented Green's functions for two-dimensional heat conduction in thin plates.

The work described here uses the BEM to define condensation areas in two-dimensional spaces, using an iterative scheme. The present iterative scheme extends the authors' earlier work on the definition of two-dimensional condensation zones across straight walls [14]. That technique is extended here to cater for curved geometries, which requires the use of quadratic curved boundary elements. The resulting model is then applied to the study of the condensation dependence on the curvature of a wall inserted between two straight walls.

Although walls are usually straight, the use of curved walls is a popular option in architecture today, which embraces the design of simple buildings and large amphitheatres and auditoriums, and so has to be considered. The effects of the curvature on the heat and moisture transfer have been overlooked in the past. The present paper aims to improve our understanding of this problem.

Initially, the BEM is used to compute the vapour and the vapour saturation pressure equilibrium across the two-dimensional element being analysed. Then the sub-domain at higher risk of condensation, i.e. where the vapour pressure is greater than the vapour saturation pressure, is identified. Using the Glaser assumptions, the vapour pressure must be equal to the vapour saturation pressure along part of this sub-domain. This is accomplished using an iterative process. First, a small region is selected inside this sub-domain, where there is a greater difference between the vapour pressure and the vapour saturation pressure. The vapour saturation pressure conditions are ascribed along the boundary of this small region, and a new vapour equilibrium is calculated, using the BEM. A second region is then selected, and the vapour pressure is again forced to be equal to the vapour saturation pressure along the boundary of this new region. A new vapour pressure equilibrium is then defined. This process is repeated until the vapour pressure across the whole domain does not exceed the vapour saturation pressure. At the end of the iterative process, the area where the vapour pressure is equal to the vapour saturation pressure is the condensation zone. Once the condensation zone has been defined, the condensed moisture is quantified.

This model thus defines the condensation across two-dimensional elements, and enables the variation of condensed vapour along the condensation zone to be quantified.

The first part of this paper states the problem and indicates the main integrals required to solve the BEM, including the necessary Green's functions. The iterative 
process used to define the condensation zone is then described, and the method for calculating the amount of condensed water is explained. A brief validation of the BEM formulation is presented, using a hollow cylinder subjected to a steady-state heat and vapour diffusion, for which an analytical solution is known. The BEM model is then used to compute the condensation zones occurring in, and the amount of liquid water generated across, uniformly thick walls with a curved section of varying curvature. The walls are subjected to constant thermal conditions.

\section{Definition of the problem}

This work aims to determine the importance of curvature on the condensation patterns across a construction element that consists of a curved segment flanked by straight segments when this element is subjected to prescribed moisture and temperature boundary conditions. The condensation zone is defined assuming that the vapour pressure at any point can never be greater than the vapour saturation pressure.

\subsection{BEM formulation}

Fig. 1 gives a schematic representation of the problem. A homogeneous two-dimensional region $(\Omega)$ with isotropic vapour permeability $\left(\pi_{\mathfrak{p}}\right)$ is bounded by a boundary $(\mathbf{S})$. Vapour pressure values $\left(P_{w}\right)$ are specified in a portion of this boundary $\left(\mathbf{S}_{1}\right)$ (Dirichlet conditions), and normal vapour fluxes are prescribed (Neumann conditions) over the remainder of $\mathrm{S}\left(\mathbf{S}_{2}\right)$. If it is assumed that no moisture sources or sinks exist inside the domain being analysed, then the steady-state vapour pressure equilibrium is governed by Laplace's equation, which has to be satisfied by $P_{w}$ [15]:

$\frac{\partial^{2} P_{w}}{\partial x^{2}}+\frac{\partial^{2} P_{w}}{\partial y^{2}}=0$

where $x$ and $y$ are the coordinates in the Cartesian coordinate system.

The corresponding vapour flux is given by Fick's law:

$\vec{g}=-\pi_{\mathrm{p}} \vec{\nabla} P_{w}$,

where $\vec{\nabla} P_{w}=\left(\partial P_{w} / \partial x\right) \vec{e}_{1}+\left(\partial P_{w} / \partial y\right) \vec{e}_{2}$ is the vapour pressure gradient.

The maximum vapour pressure that can occur at any internal point of the domain $\Omega$ is the vapour saturation pressure $\left(P_{\mathrm{S}}\right)$, which is a function of the temperature at each point (BS $5250[16]$ ),

$P_{\mathrm{S}}=610.5 \mathrm{e}^{(17.269 T / 237.3+T)}$

where $T$ is the temperature in ${ }^{\circ} \mathrm{C}$ and $P_{\mathrm{s}}$ is the vapour saturation pressure in $\mathrm{Pa}$.

According to Eq. (3), the vapour saturation pressure is dependent on the temperature. Thus, the temperature

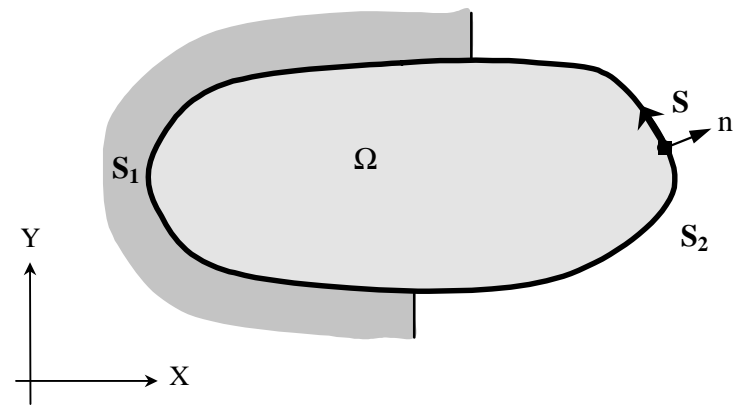

Fig. 1. Geometrical definitions for Laplace equation.

distribution must be found throughout the domain under analysis. The Laplace equation is also used to represent two-dimensional steady-state heat conduction in an isotropic region, where no internal heat sources or sinks exist,

$\frac{\partial^{2} T}{\partial x^{2}}+\frac{\partial^{2} T}{\partial y^{2}}=0$,

where $T$ is the temperature, and $x$ and $y$ define the Cartesian coordinate system used.

The heat flow is then given by Fourier's law:

$\vec{q}=-\lambda \vec{\nabla} T$,

where $\lambda$ is the material's conductivity and $\vec{\nabla} T=(\partial T / \partial x) \vec{e}_{1}+$ $(\partial T / \partial y) \vec{e}_{2}$ is the temperature gradient. The boundary conditions prescribed are the known temperatures (Dirichlet conditions) or heat fluxes (Neumann conditions) along the boundary [17].

Thus, the definition of the vapour and vapour saturation pressure across the element analysed requires the solution of a temperature and a vapour pressure equilibrium problem. This solution is obtained using the BEM, for which the boundary alone has to be discretized. As there is a considerable amount of work on the use of the BEM for solving problems of heat and moisture transfer in steady-state conditions in the literature (e.g. Manolis et al. [18]), only a brief description of the equations used for the problem stated is given here.

The boundary integral equation used as a starting point to solve this problem can be represented by

$u\left(x_{l}\right) c+\int_{S} H\left(x, x_{l}, n\right) u(x) \mathrm{d} S=\int_{S} G\left(x, x_{l}\right) q(x, n) \mathrm{d} S$,

where $c$ is a constant that equals $1 / 2$ for a smooth boundary; $u(x)$ and $q(x, n)$ are the known boundary temperatures or vapour pressures and heat or vapour fluxes, respectively; $G\left(x, x_{l}\right)$ is the Green's function component for temperature or vapour pressures, and $H\left(x, x_{l}, n\right)$ is the corresponding flux component on boundary $S$, caused by a unit point force applied at the source $x_{l}$; vector $n$ is the unit outward normal at the boundary.

The boundary must be discretized before this integral can be evaluated for an arbitrary cross-section. Therefore, in a 


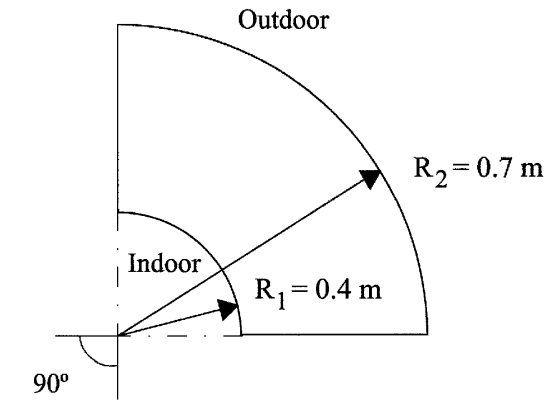

(a)

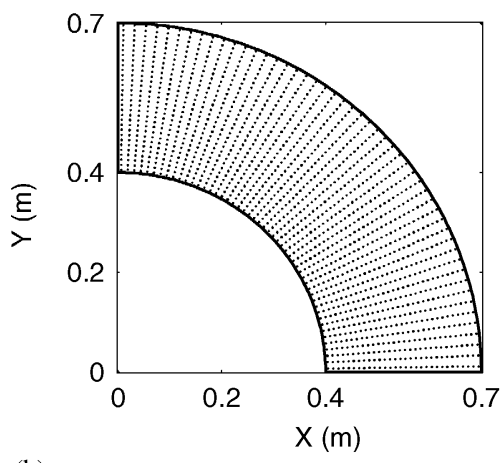

(b)

Fig. 2. Validation model: (a) Geometry of the model. (b) Grid of receivers.

situation where $N$ boundary elements are used and nodal temperature or vapour pressure and heat or vapour fluxes within each element are taken to be constant and to have the same value at the respective nodal point, Eq. (6) becomes

$\frac{1}{2} u\left(x_{l}\right)+\sum_{j=1}^{N} H^{k l} u\left(x_{k}\right)=\sum_{j=1}^{N} G^{k l} q\left(x_{k}, n_{l}\right)$,

where,

$G^{k l}=\int_{S_{k}} G\left(x_{k}, x_{l}\right) \mathrm{d} S_{k}$,

$H^{k l}=\int_{S_{k}} H\left(x_{k}, x_{l}, n_{l}\right) \mathrm{d} S_{k}$,

where $G\left(x_{k}, x_{l}\right)$ is the Green's function component for temperature or moisture; $H\left(x_{k}, x_{l}, n_{l}\right)$ is the corresponding flux component at $x_{k}$ due to a concentrated load at $x_{l}$, and $n_{l}$ is the normal outward unit for the $l$ th boundary segment $S_{k}$. The variables $u\left(x_{k}\right)$ and $q\left(x_{k}, n_{l}\right)$ can be written in terms of interpolation functions. In the present article both constant and quadratic elements are used. The constant elements are used along the straight boundaries, whereas the quadratic elements are applied to the curved boundaries of the models.

The free space Green's function assumed here is (e.g. Brebbia et al. [19]),

$G\left(x_{k}, x_{l}\right)=1 /(2 \pi \gamma) \ln (1 / r)$,

where $\gamma$ represents the thermal conductivity $(\lambda)$ or the vapour permeability $\left(\pi_{\mathrm{p}}\right)$ of the material, which is taken to be constant, and $r$ is the distance between the source and the point where the value of the function is evaluated.

The Fourier/Fick law is applied to the derivatives of $G$, in relation to the unit outward normal direction $n$, and so the flux component expressions are given by

$H\left(x_{k}, x_{l}, n_{l}\right)=-\gamma \frac{\partial G\left(x_{k}, x_{l}\right)}{\partial n}=-1 /(2 \pi) \frac{\partial \ln (1 / r)}{\partial n}$.

When the integrations given by Eqs. (8) and (9) are performed along the loaded element, the integrands exhibit a singularity. When the variables $u\left(x_{k}\right)$ and $q\left(x_{k}, n_{l}\right)$ are written in terms of a constant function, these integrations can be performed analytically [20]. When $u\left(x_{k}\right)$ and $q\left(x_{k}, n_{l}\right)$ are expressed in terms of a quadratic function, the required integrals in Eqs. (8) and (9) are composed of two parts, one with a singular term of the form $\ln (1 / \eta)$, (where $\eta$ is the local coordinate system), and the other with no singularity. The first part is integrated by means of a special integration formula of the type

$I=\int_{0}^{1} \ln \left(\frac{1}{\eta}\right) f(\eta) \mathrm{d} \eta \cong \sum A_{\mathrm{i}} f\left(x_{\mathrm{i}}\right)$,

where $A_{\mathrm{i}}$ are weights and $x_{\mathrm{i}}$ are local nodal coordinates. The second part is integrated using a standard Gaussian quadrature formula. When the integration is not along the loaded element the integrations again use a standard Gaussian quadrature scheme.

If we assume that the position of the load can be applied in sequence along all nodes, while ascribing the required boundary conditions, a system of equations can be built up. This system is solved for the nodal temperature or vapour pressure and for the heat or moisture flows. When these values are determined, it is possible to compute the values for the temperature or vapour pressure variable, or their derivatives at any point within the domain.

\subsection{Illustration and validation of the iterative process}

Consider a hollow cylinder with inner radius $r_{1}\left(r_{1}=\right.$ $0.4 \mathrm{~m})$ and outer radius $r_{2}\left(r_{2}=0.7 \mathrm{~m}\right)$ as in Fig. 2a, made of cellular autoclaved concrete $\left(\lambda=0.16 \mathrm{~W}^{\circ} \mathrm{C}^{-1} \mathrm{~m}^{-1}, \pi=\right.$ $25 \times 10^{-12} \mathrm{Kg} \mathrm{m}^{-1} \mathrm{~s}^{-1} \mathrm{~Pa}^{-1}$ ). The inner and outer surface conditions are given in Table 1. For a steady-state condition, with no heat generation, the temperature distribution is given by the following equation:

$T(r)=\frac{T_{1}-T_{2}}{\ln \left(r_{1} / r_{2}\right)} \ln \left(\frac{r}{r_{2}}\right)+T_{2}$,

where $r$ is the distance from the axis of the cylinder. 
Table 1

Boundary conditions (validation)

\begin{tabular}{llll}
\hline $\begin{array}{l}\text { Indoor } \\
\text { temperature }\end{array}$ & $\begin{array}{l}\text { Indoor relative } \\
\text { humidity }\end{array}$ & $\begin{array}{l}\text { Outdoor } \\
\text { temperature }\end{array}$ & $\begin{array}{l}\text { Outdoor relative } \\
\text { humidity }\end{array}$ \\
\hline $18.0^{\circ} \mathrm{C}$ & $90 \%$ & $0.0^{\circ} \mathrm{C}$ & $85 \%$ \\
$P \mathrm{~s}_{\mathrm{i}}=2062.83 \mathrm{~Pa}$ & $P \mathrm{~s}_{\mathrm{e}}=610.5 \mathrm{~Pa}$ \\
$P w_{\mathrm{i}}=1856.55 \mathrm{~Pa}$ & $P w_{\mathrm{e}}=518.93 \mathrm{~Pa}$ \\
\hline
\end{tabular}

Given the geometrical symmetry of the problem, only a quarter of the hollow cylinder is modelled. Moisture diffusion is simulated by ascribing null vapour flow across the radial boundaries of the cylinder.

Two fictitious layers, one internal $\left(\lambda=0.1667 \mathrm{~W}^{\circ} \mathrm{C}^{-1} \mathrm{~m}^{-1}\right)$ and the other external $\left(\lambda=0.5 \mathrm{~W}^{\circ} \mathrm{C}^{-1} \mathrm{~m}^{-1}\right)$, each $0.02 \mathrm{~m}$ thick, are added to the two faces of the hollow cylinder to simulate the internal and the external thermal surface resistance $\left(1 / h_{\mathrm{i}}=0.12 \mathrm{~m}^{2}{ }^{\circ} \mathrm{CW}^{-1}\right.$ and $\left.1 / h_{\mathrm{e}}=0.04 \mathrm{~m}^{2}{ }^{\circ} \mathrm{CW}^{-1}\right)$, respectively. This procedure is frequently adopted in Civil Engineering, since it allows the radiation and convection contributions to be taken into account [21]. The thicknesses of these layers were defined so as to guarantee the internal and the external thermal surface resistance values, and to avoid the existence of a very thin layer, which would lead to a larger number of boundary elements. Note that the length of the boundary elements should be at least 4 times smaller than the thickness of the layer to guarantee that the BEM performs well. It should be further mentioned that there is no vapour resistance associated with these layers.

The BEM model is first used to compute the temperature distribution across the hollow cylinder over a fine grid of internal points (see Fig. 2b) spaced at equal radial distances from one another of $0.01 \mathrm{~m}$. Two hundred elements are used to discretize each curved boundary, and one hundred and fifty elements discretize each radial boundary. Eq. (3) is then used to calculate vapour saturation pressure distribution $\left(P_{\mathrm{s}}\right)$. The first vapour pressure $\left(P_{w}\right)$ along the grid of internal points is calculated, imposing the known vapour pressure conditions at the boundaries. The initial distribution of $P_{w}$ and $P_{\mathrm{s}}$ are displayed in Fig. 3. If at this stage, all internal points in the grid register a vapour pressure lower than $P_{\mathrm{s}}$, then this is the final vapour pressure distribution, and no condensation exists. In the present example, there are internal points recording $P_{w}$, which is greater than $P_{\mathrm{s}}$, meaning that condensation will occur within a region inside this sub domain, labelled $C_{\text {risk }}$ in Fig. 3.

The internal point whose estimated vapour pressure most exceeds the corresponding saturation pressure is identified. A small region is defined around this internal point. In the present example, this small region is a cell made of two straight boundaries and two curved arcs, forming a segment of a circular hollow. The length of the straight segments is equal to the radial distance between internal points and an azimuthal distance, equal to that between the internal points,

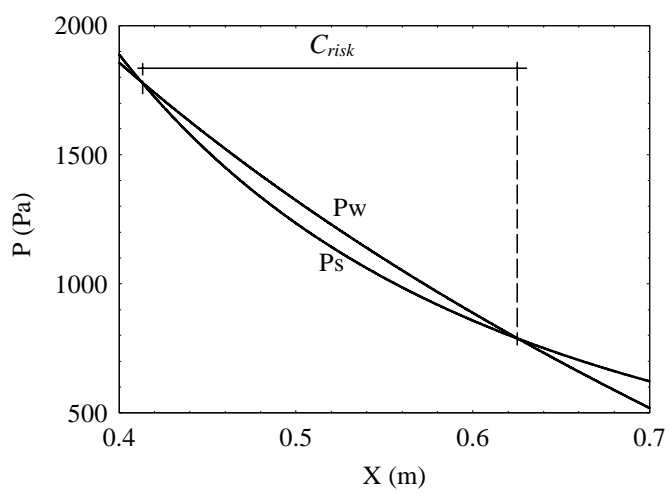

Fig. 3. Initial vapour equilibrium: vapour saturation pressure distribution $\left(P_{\mathrm{s}}\right)$ versus first vapour pressure estimation $\left(P_{w}\right)$.

separates these straight segments. The difference between the radii of the two curved arcs is equal to length of the straight segments. This cell is centred at the selected internal point (see Fig. 4a). The boundary of this small region is then discretized with 4 constant boundary elements, placed on each straight segment while each curved arc is discretized with 4 quadratic curved boundary elements. Different simulations were performed with varying numbers of boundary elements being used to discretize the boundary. It was concluded that the difference in the responses was negligible for more than four elements (less than $1 \%$ of the total liquid water computed). The vapour saturation pressure is ascribed at the nodes of the boundary elements used to discretize the small region. The exterior boundary conditions of the wall are kept constant along this process. A new vapour pressure equilibrium is then computed. Fig. $4 \mathrm{~b}$ displays the new difference between $P_{\mathrm{s}}$ and $P_{w}$. This iterative process is repeated until no internal point registers a $P_{w}$ above $P_{\mathrm{s}}$. At each iteration, the new boundary elements are checked to see if any of them coincide with a previous one. If this situation occurs, then both elements are removed, because the distribution of $P_{w}$ is being calculated outside the sub-domains created by the small regions, which is the area where additional small regions may be subject to condensation. Fig. 5 displays the results obtained at the end of iteration 110 , which illustrates this procedure.

The final results, after all the iterations have been run, are given in Fig. 6. This figure indicates the existence of a condensation area, labelled $C_{\text {Final }}$, located between coordinates $r_{1}=0.440 \mathrm{~m}$ and $r_{2}=0.540 \mathrm{~m}$. The analytical definition of the condensation zone leads to $r_{1}=0.447 \mathrm{~m}$ and $r_{2}=0.544 \mathrm{~m}$. The results confirm a very good approximation of the calculated values obtained by the iterative process. The precision of the numerical solution would improve if finer regions were used. It should be noted that this iterative process requires the use of different subdomains, which need to be discretized with boundary elements, making this algorithm less attractive in terms of computational cost. The method would improve if other criteria were used to 

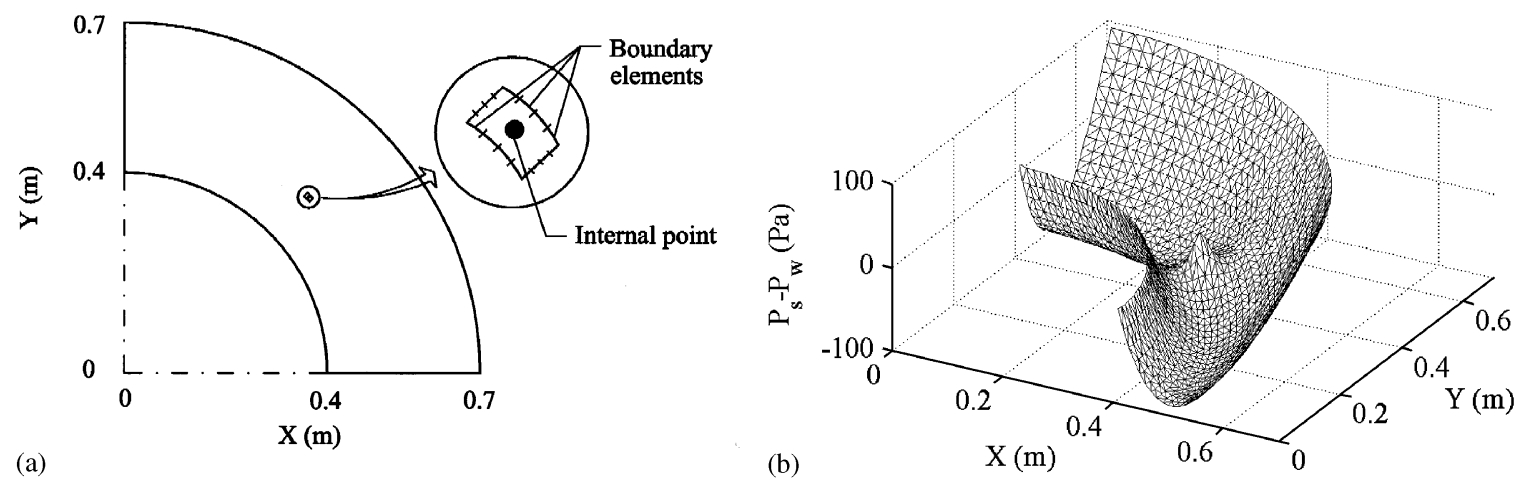

Fig. 4. Results after iteration 1. (a) Cell around the selected internal point. (b) $P_{\mathrm{s}}-P_{w}$ distribution.
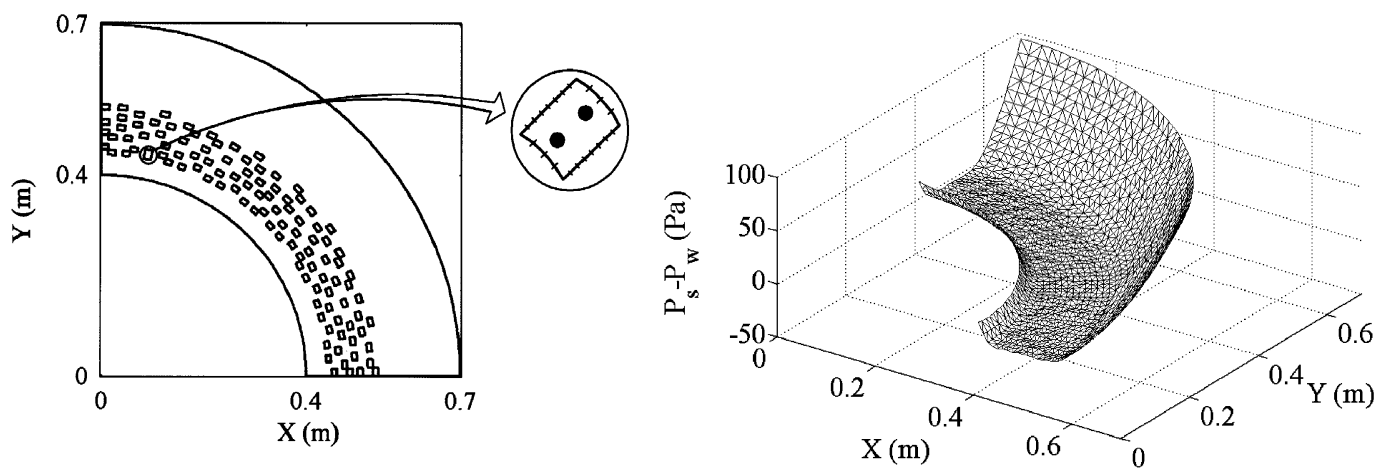

Fig. 5. Results after iteration 110. (a) Selected internal points after iteration 110. (b) $P_{\mathrm{s}}-P_{w}$ after iteration 110.

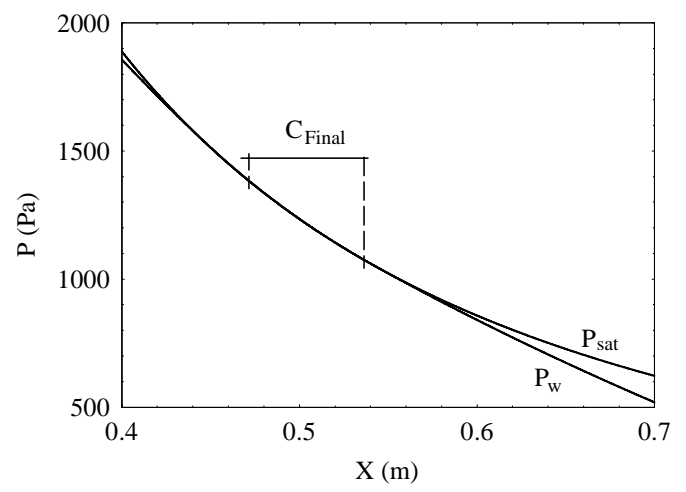

Fig. 6. Results after the final iteration: vapour saturation pressure distribution $P_{\mathrm{s}}$ versus final vapour pressure estimation $P_{w}$.

define more general subdomains where condensation occurs, that is, if each iteration identified more than one receiver. The development of these criteria will be object of a new research project.

Once the area of condensation has been defined, the BEM is next applied to compute the moisture fluxes within each small region (small hollow segment). Vapour saturation pressure conditions are ascribed to each boundary element, and vapour fluxes are computed using the BEM.
Neighbouring cells may have distinct vapour fluxes across common elements. The amount of liquid water produced within each cell is quantified by the summation of these flux jumps along the entire cell boundary. The total liquid water in the present example was found to be $122 \mathrm{mg} \mathrm{h}^{-1}$, which is close to the analytical value $117 \mathrm{mg} \mathrm{h}^{-1}$.

As stated above, the method proposed here is based on the Glaser approach. The moisture transfer is assumed to be pure water vapour diffusion, the thermal conductivity is assumed to be constant and the specific heat capacity of the materials is considered to be not relevant.

In fact, thermal conductivity depends on the moisture content, and heat is released by condensation. This will change the temperature distribution and vapour saturation pressure values, and so affect the amount of condensation. However, the amount of condensation is overestimated using this approach, which may be regarded as useful for design purposes [22], and it is, after all, recommended by the DIN 4108 [5] and prEN ISO 13788 [6] standards.

\section{Steady-state heat and moisture diffusion in curved walls}

A homogeneous wall, $0.30 \mathrm{~m}$ thick, is used to analyse the change in condensation patterns between a straight and 


\begin{tabular}{|c|c|c|c|}
\hline \multirow{2}{*}{} & & $\mathrm{R}_{1}(\mathrm{~m})$ & $\mathrm{R}_{2}(\mathrm{~m})$ \\
\cline { 2 - 4 } & Model 1 & 0.0 & 0.3 \\
\cline { 2 - 4 } & Model 2 & 0.4 & 0.7 \\
\hline & Model 3 & 0.8 & 1.1 \\
\hline
\end{tabular}

Fig. 7. Curved wall section geometries.

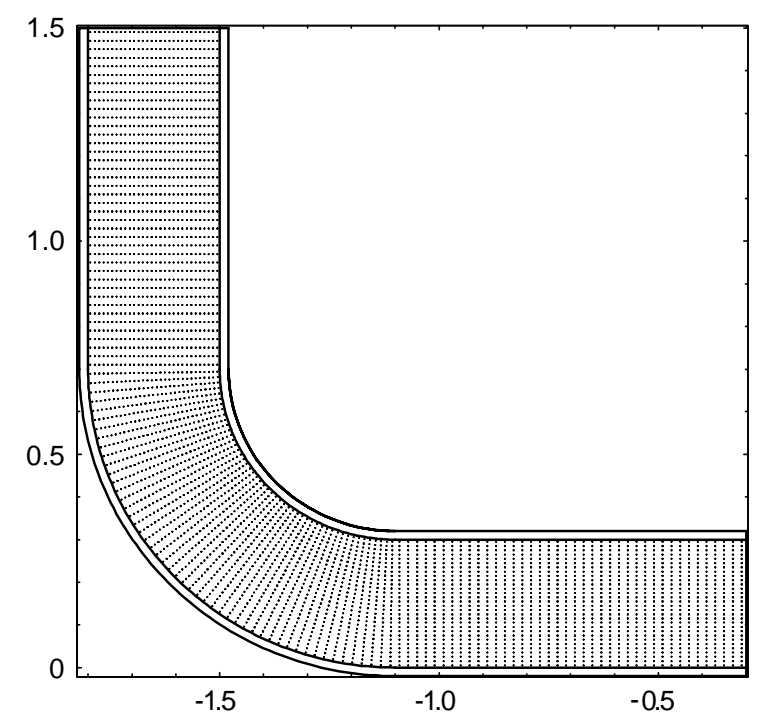

Fig. 8. Grid of internal points (Model 2).

a curved wall. To illustrate the difference in thermal behaviour, a section of a curved wall is placed between two perpendicular straight walls. Different radii were considered for the curved wall. Fig. 7 illustrates the geometries (Models 1-3) of the curved wall sections used. The length of the straight part of the wall was defined in such a way that, the vapour and heat fluxes at the two extremities of the model's walls have a one-dimensional behaviour; that is, the diffusions at these points are orthogonal to the wall boundaries.

Fig. 8 illustrates one of the internal point grids (3900 internal points) used to compute the temperature and vapour pressure distribution across the wall (Model 2). The BEM uses constant elements and the boundary is approximated by a polygon. Nine hundred and twenty four elements are used to model the problem described here. During the iterative process, each new cell is modelled with 16 boundary elements.

The first set of examples illustrates the case where condensation occurs in the vicinity of the wall surface, while a second application gives the results when the condensation phenomenon occurs inside the wall.

\subsection{Condensation in the vicinity of the wall surface}

The present example models a concrete wall. The thermal conductivity of the wall is taken to be $1.75 \mathrm{~W}^{\circ} \mathrm{C}^{-1} \mathrm{~m}^{-1}$, while the vapour permeability is $6.30 E-12 \mathrm{Kg} \mathrm{Pa}^{-1}$ $\mathrm{s}^{-1} \mathrm{~m}^{-1}$. As before, two fictitious layers are added to simulate the internal and the external thermal surface resistance $\left(1 / h_{\mathrm{i}}=0.12 \mathrm{~m}^{2}{ }^{\circ} \mathrm{CW}^{-1}\right.$ and $\left.1 / h_{\mathrm{e}}=0.04 \mathrm{~m}^{2}{ }^{\circ} \mathrm{CW}^{-1}\right)$ [21]. Null fluxes are ascribed to the boundary perpendicular to the wall surfaces at the two extremities of the model's walls.

Two different moisture and thermal boundary conditions are assumed, when the wall is straight, as listed in Table 2. The first leads to the occurrence of condensation (Case 1), while the second does not allow condensation to occur (Case 2). Two situations arise for each of these two Cases; Situation 1, where the indoor thermal and moisture conditions are prescribed along the smaller perimeter surface of the construction element and Situation 2, where the indoor hygrothermal conditions are ascribed to the surface with larger perimeter. The characteristics of the external and internal environmental selected are common in Portugal, leading to different types of pathology.

The temperature and vapour pressure distribution across the wall are computed using the BEM model. After the saturation pressure distribution has been defined by Eq. (3), the iterative process described above is performed, to define the condensation patterns and the amount of liquid water. In this calculation, the moisture fluxes on the external surface elements are held to be null if condensation occurs at a cell adjacent to the surface. Another condition may be easily incorporated into the model, such as the existence of a fictitious layer to simulate the permeability surface resistance. The condensation zone would remain the same for all conditions, but the amount of liquid water generated at the surface would be smaller.

Fig. 9 shows the space domains where the calculated saturated pressures are lower than the computed vapour pressures, when the wall is straight (Cases 1 and 2). A grey scale is used in these plots to illustrate the amplitude differences between these two curves. The darker colours (bigger amplitude values) indicate the areas where there is a greater risk of condensation occurring. As mentioned above, the wall under the boundary conditions prescribed for Case 1 shows condensation appearing on its interior face. However, the wall under the boundary conditions prescribed for Case 2 seems to be free of condensation. In addition, these plots identify the final area of condensation (bounded by solid lines) defined by the iterative process. It can be seen that the condensation zone is smaller than the area where the vapour pressure exceeded the vapour saturation pressure at the beginning of the iterative process. This behaviour is due to the new vapour pressure equilibrium defined by the iteration processes.

The curvature radii of the walls take the values listed in Fig. 7. As the radius of the wall curvature increases, the 
Table 2

Boundary conditions (Cases 1 and 2)

\begin{tabular}{llll}
\hline & $\begin{array}{l}\text { Indoor } \\
\text { temperature }\end{array}$ & $\begin{array}{l}\text { Indoor relative } \\
\text { humidity }\end{array}$ & $\begin{array}{l}\text { Outdoor relative } \\
\text { humidity } \\
\text { temperature }\end{array}$ \\
\hline Case 1 & $20.0^{\circ} \mathrm{C}$ & $70 \%$ & $0.0^{\circ} \mathrm{C}$ \\
$P_{w}=549.5 \mathrm{~Pa}$ & $90 \%$ \\
Case 2 & $P_{w}=1635.9 \mathrm{~Pa}$ & & $0.0^{\circ} \mathrm{C}$ \\
& $14.0^{\circ} \mathrm{C}$ & $70 \%$ & $549.5 \mathrm{~Pa}$ \\
\hline
\end{tabular}
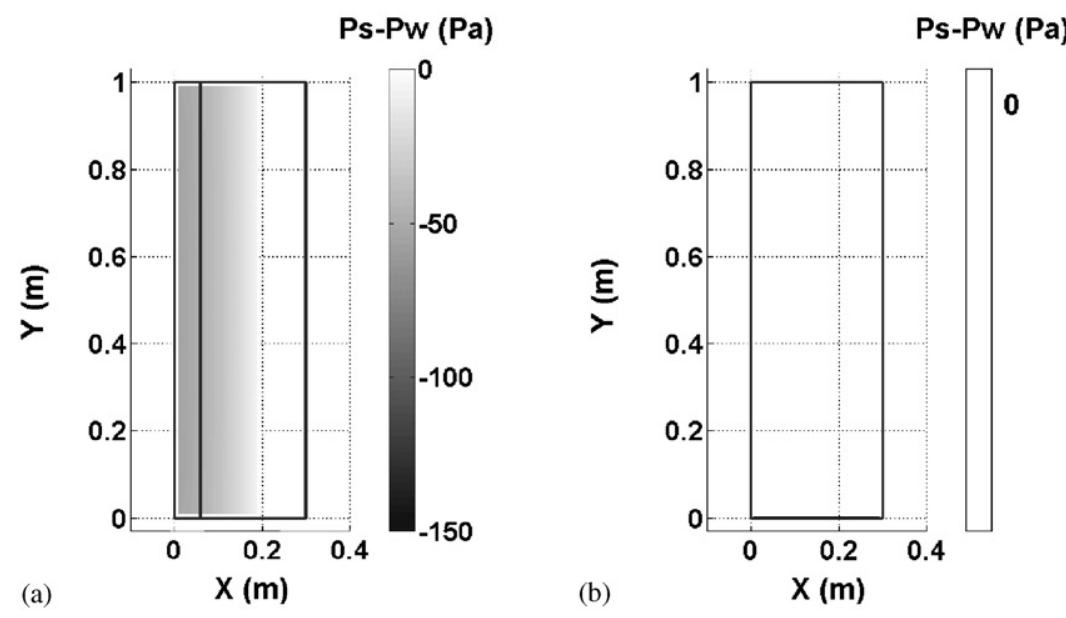

Fig. 9. Condensation patterns for straight walls. (a) Case 1 (b) Case 2.

calculated results exhibit similar condensation patterns to those seen in the case of the straight wall.

Fig. 10 illustrates both the difference between the vapour saturation pressure and the vapour pressure after the first iteration (grey scale), and the final condensation patch (bounded by solid lines). When the wall is built with a narrower curve, and indoor hygrothermal conditions are ascribed to the side of the wall with smaller perimeter (Situation 1), the risk of condensation increases. This can be seen in Fig. 10, which shows that the difference between $P_{\mathrm{s}}$ and $P_{w}$ increases as radii are reduced. However, the condensation area predicted by the iterative process reveals the existence of a narrow patch of condensation in the vicinity of the interior corner of the wall. As the distance from the corner increases, the final condensation area approaches that found for the straight wall. It can further be observed that when Situation 2 is modelled, the patch of condensation across the curved section of the wall diminishes as the radius of the curve increases. As the radius of the curved wall increases, the hygrothermal behaviour resembles that found for the straight wall model. Additional simulations, not detailed here, showed that it would require a radius of $7.5 \mathrm{~m}$ before behaviour similar to that of a straight wall was observed for Situation 1 , while a radius of $35 \mathrm{~m}$ is needed when Situation 2 is modelled.
This is because, as the radius of the curved wall section decreases (Situation 1), the ratio between the heated, interior, surface and the cooler, exterior, surface areas also decreases. In Situation 2, the ratio between the areas of heated and unheated surface increases as the curved wall gets narrower.

Fig. 11 displays the amount of liquid water generated for Case 1, under Situation 1. A larger quantity of condensed moisture is concentrated in the vicinity of the inner surface, because the moisture fluxes on the external surface elements are held to be null. The cells on the curved wall have less liquid water compared with the sub-domains of the straight walls. This is because the flux jumps between adjacent cells are smaller along the curved section of the wall.

Fig. 12 shows the condensation patterns registered for the wall geometries described above, when subjected to the hygrothermal conditions prescribed for Case 2 . The results show behaviour similar to that found in the previous case. However, it is important to notice that in this case the straight wall geometry does not lead to condensation, whereas the curved wall under the hygrothermal conditions prescribed by Situation 1 does. Fig. 12 also illustrates the amount of liquid water generated by Model 3. It can be seen that, inside the curved section of the wall, the amount of condensed moisture 

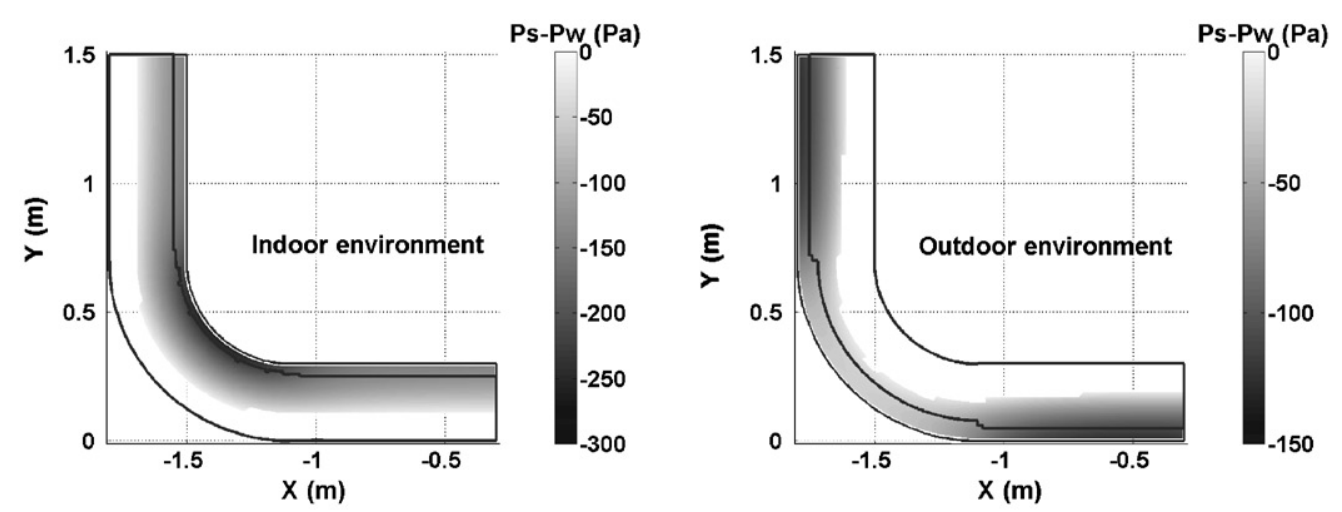

Model $2\left(\mathrm{R}_{1}=0.4 \mathrm{~m} ; \mathrm{R}_{2}=0.7 \mathrm{~m}\right)$
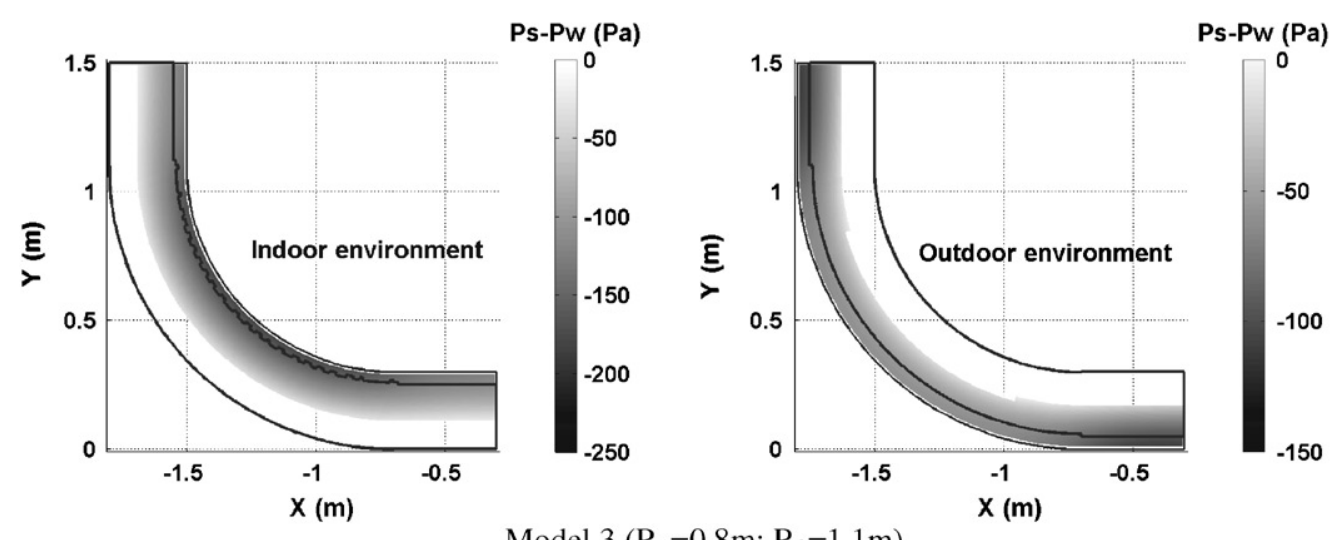

Model $3\left(\mathrm{R}_{1}=0.8 \mathrm{~m} ; \mathrm{R}_{2}=1.1 \mathrm{~m}\right)$

Fig. 10. Condensation patterns for Case 1 (Situations 1 and 2).

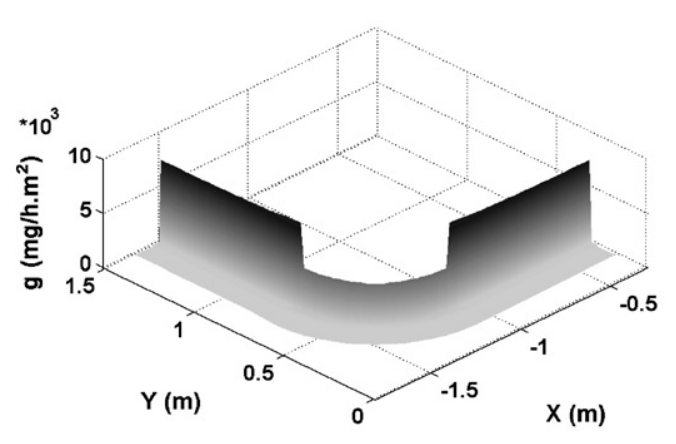

Model $2\left(\mathrm{R}_{1}=0.4 \mathrm{~m} ; \mathrm{R}_{2}=0.7 \mathrm{~m}\right)$

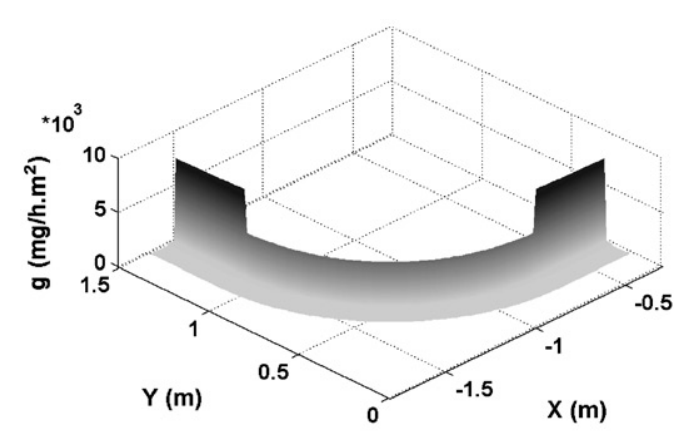

Model $3\left(\mathrm{R}_{1}=0.8 \mathrm{~m} ; \mathrm{R}_{2}=1.1 \mathrm{~m}\right)$

Fig. 11. Amount of liquid water generated for Case 1 (Situation 1).

is nearly constant, whereas along the straight sections of the wall, it vanishes.

\subsection{Condensation inside the wall}

In the following example, the wall is made from cellular autoclaved concrete, whose thermal conductivity and vapour permeability parameters are $0.16 \mathrm{~W}^{\circ} \mathrm{C}^{-1} \mathrm{~m}^{-1}$ and $25 \times 10^{12} \mathrm{Kg} \mathrm{m}^{-1} \mathrm{~s}^{-1} \mathrm{~Pa}^{-1}$, respectively. The fictitious layers, added to the wall model to simulate the internal and the external thermal surface resistance, remain unchanged $\left(1 / h_{\mathrm{i}}=0.12 \mathrm{~m}^{2}{ }^{\circ} \mathrm{CW}^{-1}\right.$ and $\left.1 / h_{\mathrm{e}}=0.04 \mathrm{~m}^{2}{ }^{\circ} \mathrm{CW}^{-1}\right)$. Again, null fluxes are ascribed to the boundary perpendicular to the wall surfaces at the two extremities of the model's walls. The hygrothermal boundary conditions are listed in Table 3. Again, the external and internal hygrothermal conditions are representative of the environment in Portugal.

Fig. 13 illustrates the risk of a condensation zone under the conditions of Situation 2, when the construction element is subjected to the boundary conditions given in Table 3 . 


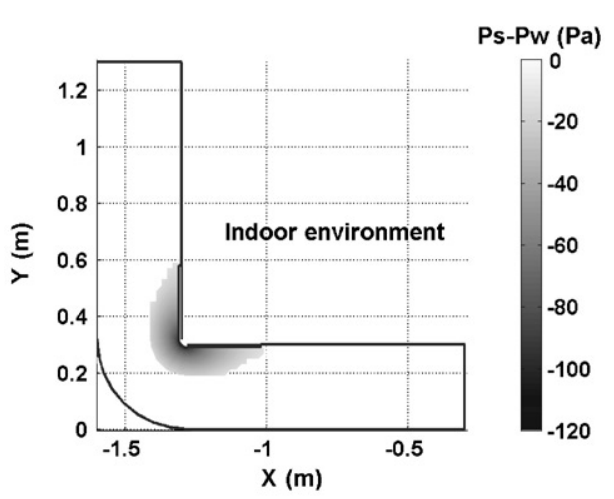

Model $1\left(\mathrm{R}_{1}=0.0 \mathrm{~m} ; \mathrm{R}_{2}=0.3 \mathrm{~m}\right)$

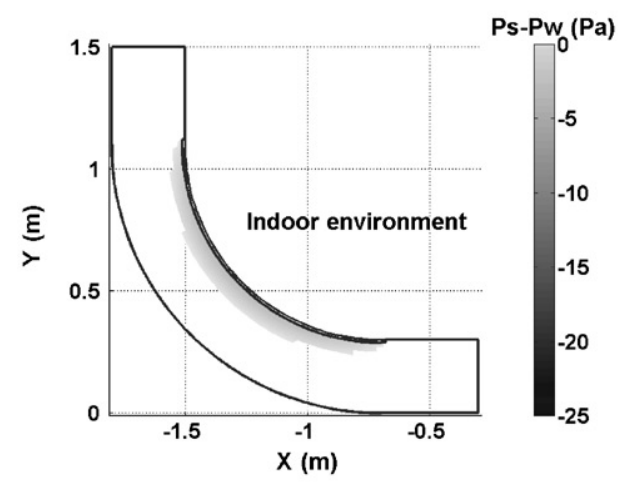

Model $3\left(\mathrm{R}_{1}=0.8 \mathrm{~m} ; \mathrm{R}_{2}=1.1 \mathrm{~m}\right)$

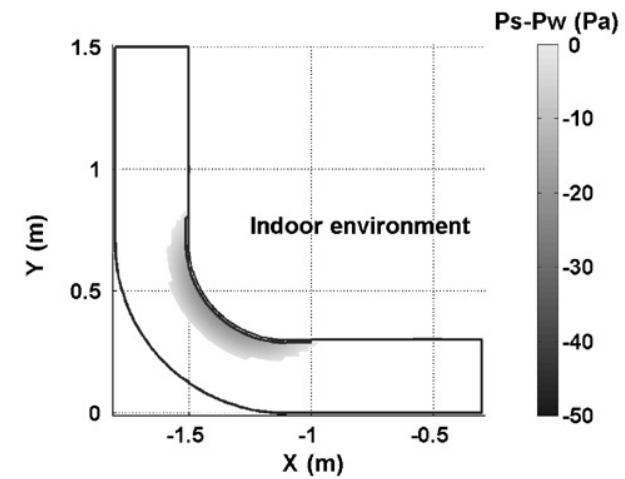

Model $2\left(\mathrm{R}_{1}=0.4 \mathrm{~m} ; \mathrm{R}_{2}=0.7 \mathrm{~m}\right)$

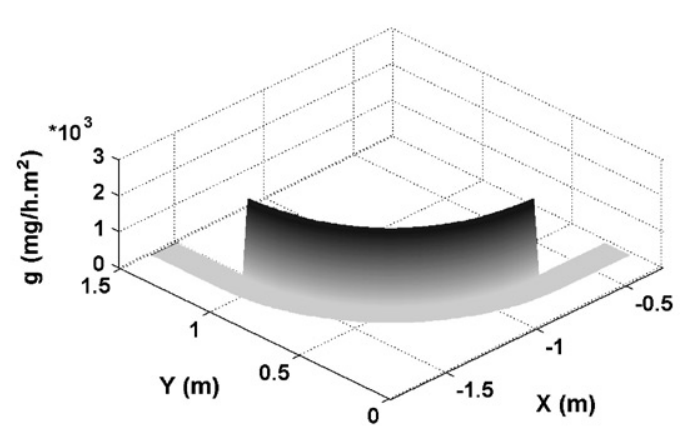

Model $3\left(\mathrm{R}_{1}=0.8 \mathrm{~m} ; \mathrm{R}_{2}=1.1 \mathrm{~m}\right)$

Fig. 12. Condensation patterns and amount of liquid water for Case 2, Situation 1.

Table 3

Boundary conditions (Case 3 )

\begin{tabular}{lllll}
\hline & $\begin{array}{l}\text { Indoor } \\
\text { temperature }\end{array}$ & $\begin{array}{l}\text { Indoor relative } \\
\text { humidity }\end{array}$ & $\begin{array}{l}\text { Outdoor } \\
\text { temperature }\end{array}$ & $\begin{array}{l}\text { Outdoor relative } \\
\text { humidity }\end{array}$ \\
\hline Case 3 & $18.0^{\circ} \mathrm{C}$ & $85 \%$ & $0.0^{\circ} \mathrm{C}$ & $90 \%$ \\
& $P_{w}=1753.4 \mathrm{~Pa}$ & & $P_{w}=549.5 \mathrm{~Pa}$ & \\
\hline
\end{tabular}

The results reveal an absence of condensation in the curved section of the wall when its radius is smaller (see Model 1). As the radius of the curved section of the wall increases, the condensation patches approach the result computed for the straight wall. However, the radius of the curved wall needs to be very large for the behaviour to be similar to that found for the straight wall. The amount of liquid water obtained for Model 3 is also given in Fig. 13. It can be seen that larger amounts of condensed water are generated in the transition zone between the curved and the straight sections, due to the difference in the size of the areas of condensation. At these specific points, the two-dimensional behaviour of the fluxes leads to a larger amount of condensed moisture.

Fig. 14 displays the condensation patterns registered for the same wall geometries, when the hygrothermal conditions are those prescribed for Situation 1. The results indicate a larger condensation patch in the vicinity of the curved section of the wall. As the curvature of the wall decreases, the results once again approach those for the straight wall.

Additional simulations for both situations, not given in the paper, showed that it would require a radius of $30.0 \mathrm{~m}$ before behaviour comparable to that of a straight wall occurred.

\section{Conclusions}

The Boundary Elements Method (BEM) has been formulated and implemented to solve two-dimensional steady-state thermal conduction and vapour diffusion problems efficiently. After validation, the BEM method was used to analyse the condensation patterns of a curved wall. The results suggest that the use of one-dimensional 


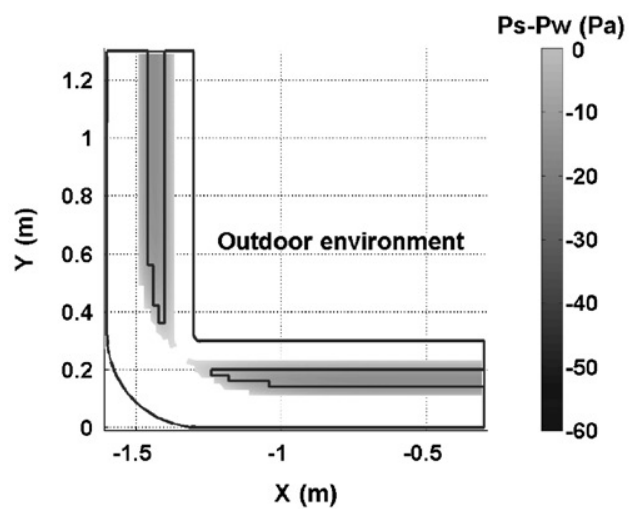

Model $1\left(\mathrm{R}_{1}=0.0 \mathrm{~m} ; \mathrm{R}_{2}=0.3 \mathrm{~m}\right)$

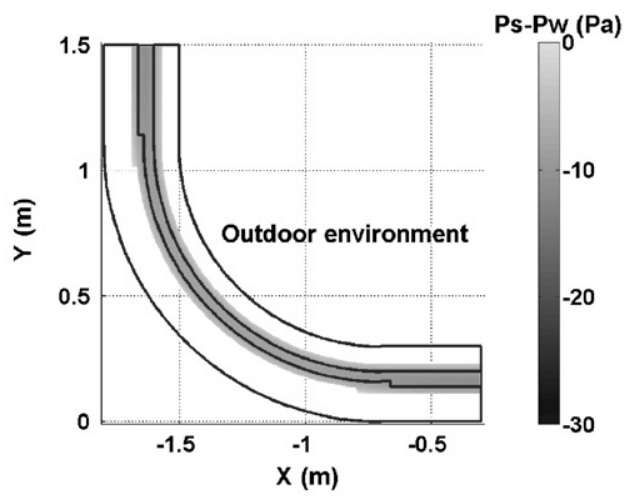

Model $3\left(\mathrm{R}_{1}=0.8 \mathrm{~m} ; \mathrm{R}_{2}=1.1 \mathrm{~m}\right)$

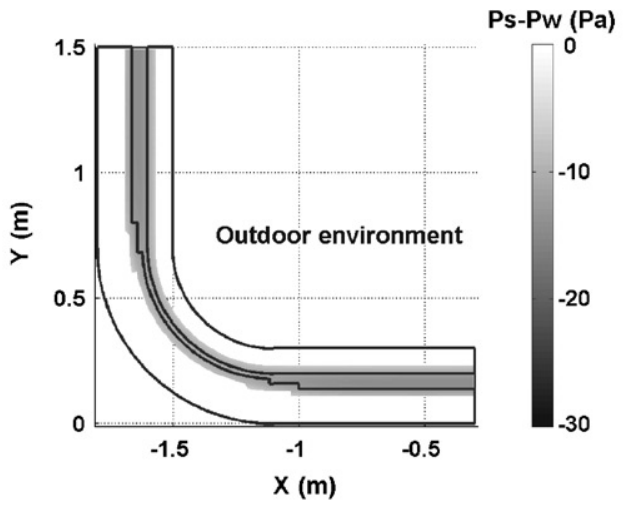

Model $2\left(\mathrm{R}_{1}=0.4 \mathrm{~m} ; \mathrm{R}_{2}=0.7 \mathrm{~m}\right)$

Fig. 13. Condensation patterns and amount of liquid water for Case 3, Situation 2.

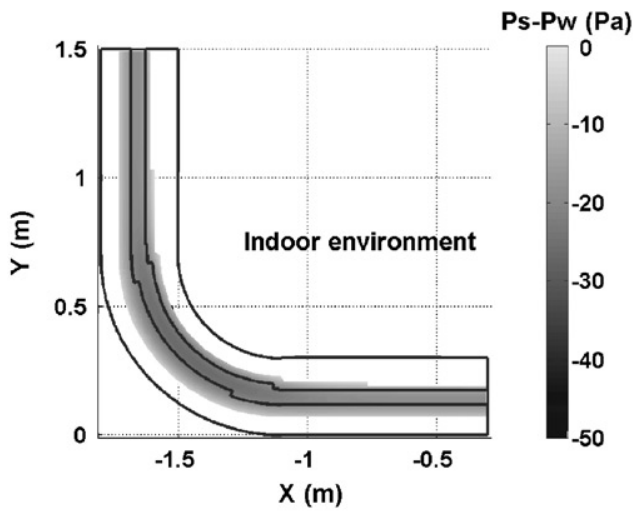

Model $2\left(\mathrm{R}_{1}=0.4 \mathrm{~m} ; \mathrm{R}_{2}=0.7 \mathrm{~m}\right)$

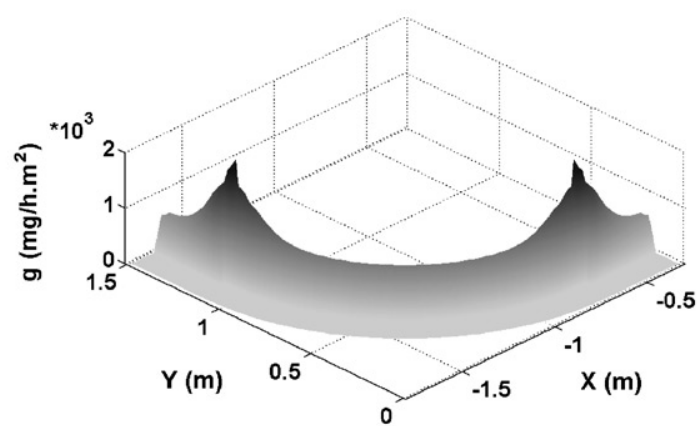

$\operatorname{Model} 3\left(\mathrm{R}_{1}=0.8 \mathrm{~m} ; \mathrm{R}_{2}=1.1 \mathrm{~m}\right)$ 


\section{References}

[1] Glaser H. Vereinfachte Berechnung der Dampfdiffusion durch geschichtete Wände bei Ausscheidung von Wasser und Eis, Kältetechnik 1958; 10 (11):358-64 and (12):386-90.

[2] Krischer O, Kast W. Die wissenschaftlichen Grundlagen der Trocknungstechnik, Dritte Auflage. Berlin: Springer, 1978.

[3] Luikov AV. Systems of differential equations of heat and mass transfer in capillary-porous bodies. International Journal of Heat and Mass Transfer 1975;18:1-4.

[4] Philip JR, De Vries DA. Moisture movement in porous materials under temperature gradients. Transactions American Geophysical Union 1957;2:222-32.

[5] DIN 4108-Wärmeschutz im Hochbau. DIN Deutsches Institut für Normung, 1981

[6] GTR WI 46 and CEN/TC 89 WI29. prEN ISO 13788-Hygrothermal performance of building components and building elements. Internal surface temperature to avoid critical surface humidity and interstitial condensation. Calculation methods. The International Organization for Standardization and European Committee for Standardization, 1999.

[7] Bathe KJ. Numerical methods in Finite Element analysis. Englewood Cliffs, NJ: Prentice-Hall, 1976.

[8] Freitas VP, Abrantes V, Crausse P. Moisture migration in building walls. Analysis of the interface phenomena. Building and Environment 1996;31(2):99-108.

[9] Mingfang T, Qigao C. Numerical simulation of thermal performance of a square column. Building and Environment 1997;32(5):411-5.

[10] Fratantonio M, Rencis JJ. Exact boundary element integrations for two-dimensional Laplace equation. Engineering Analysis with Boundary Elements 2000;24(4):325-42.

[11] Kassab AJ, Divo E. A generalized boundary integral equation for isotropic heat conduction with spatially varying thermal conductivity.
Engineering Analysis with Boundary Elements 1996;18(4): 273-86.

[12] Ochiai Y, Sekiya T. Steady-state heat conduction analysis by improved multiple-reciprocity Boundary Element Method. Engineering Analysis with Boundary Elements 1996;18(2):111-7.

[13] Melnikov YA. An alternative construction of Green's function for the two-dimensional heat conduction. Engineering Analysis with Boundary Elements 2000;24(6):467-75.

[14] Simões N, Branco F, Tadeu A. Definition of two-dimensional condensation via BEM, using the Glaser method approach. Engineering Analysis with Boundary Elements, accepted.

[15] Incropera FP, DeWitt DP. Fundamentals of heat and mass transfer, 4th ed. New York: Wiley, 1996.

[16] British Standard code of practice for control of condensation in buildings-BS 5250. British Standards Institute, UK, 1989.

[17] París F, Cañas J. Boundary Element Method. Fundamentals and applications. Oxford: University Press, 1997.

[18] Manolis GD, Beskos DE. Boundary Element Methods in elastodynamics. London: Unwin Hyman (sold to Chapman \& Hall), 1988.

[19] Brebbia CA, Dominguez J. Boundary Elements, an introductory course. Boston: Computational Mechanics Publications. New York, Boston, McGraw Hill Book Company, 1989.

[20] Hess JL, Smith AM. Calculation of potential flow about arbitrary bodies. In: Kucheman D, editor. Progress in Aeronautical Science, vol. 8. New York: Pergamon, 1967. p. 1-138.

[21] Künzel HM. Simultaneous heat and moisture transport in building components. One and two-dimensional calculation using simple parameters. Frauhnofer-Informationzentrum Raum und Bau. Stuttgart: IRB-Verlag, 1995.

[22] der Kooi JV, Moisture transport in cellular concrete roofs. PhD thesis. Eindhoven University of Technology, Waltman, Delft, 1971. 